\title{
France. Une autonomie inachevée
}

Études de cas

\section{Cécile Brennan-Sardou}

\section{(2) OpenEdition} Journals

Édition électronique

URL : https://journals.openedition.org/ries/1295

DOI : 10.4000/ries. 1295

ISSN : 2261-4265

Éditeur

France Education international

Édition imprimée

Date de publication : 1 décembre 2005

Pagination : 79-82

ISSN : 1254-4590

Référence électronique

Cécile Brennan-Sardou, « France. Une autonomie inachevée », Revue internationale d'éducation de Sèvres [En ligne], 40 | décembre 2005, mis en ligne le 10 avril 2012, consulté le 07 juillet 2021. URL : http://journals.openedition.org/ries/1295; DOI : https://doi.org/10.4000/ries.1295

Ce document a été généré automatiquement le 7 juillet 2021

(c) Tous droits réservés 


\title{
France. Une autonomie inachevée
}

\author{
Études de cas
}

\author{
Cécile Brennan-Sardou
}

1 La France est marquée par un processus global de territorialisation des politiques éducatives : projets de zone d'éducation prioritaire, projets éducatifs locaux, contrats éducatifs locaux, comités locaux de sécurité et de prévention de la délinquance, insérés dans des «contrats de ville», «d'agglomération», "d'objectifs»... À la fois « communauté particulière » et élément du système éducatif national, l'établissement scolaire est marqué par la tension entre la recherche des réponses les plus adaptées aux besoins spécifiques du terrain, à l'échelle locale, et le respect des orientations nationales pour une école républicaine marquée par l'équité et la solidarité. L'autonomie de l'établissement scolaire en France est inscrite juridiquement dans le décret du 30 août 1985; il stipule que l'établissement dispose de la personnalité juridique, d'un budget propre, d'organes assurant sa direction et son administration, et de pouvoirs de décision dans des domaines définis. Elle s'exerce, selon l'article 2, dans les domaines, majoritairement pédagogiques, suivants :

- organisation en classes et groupes d'élèves, modalités de répartition des élèves ;

- emploi des dotations en heures d'enseignement à disposition de l'établissement ;

- organisation du temps scolaire et modalités de la vie scolaire ;

- préparation de l'orientation et de l'insertion sociale et professionnelle des élèves ;

- actions de formation complémentaire et formation continue ;

- ouverture de l'établissement sur son environnement ;

- choix de sujets d'études spécifiques à l'établissement ;

- activités facultatives.

2 Les établissements utilisent-ils ces espaces d'autonomie de façon créative ? Ou bien ontils tendance à reproduire des schémas connus et éprouvés, de sorte de respecter et créer de fait une norme intériorisée ? Sans doute le fonctionnement réel se situe-t-il entre ces deux pôles.

Quels sont les établissements où l'emploi du temps est d'abord élaboré pour les élèves et non pour les professeurs? Est-il conçu dans le but de favoriser le travail d'équipe? Autorise-t-elle à une mobilité qui casse le cadre d'une répartition hebdomadaire 
répétitive, comme peuvent y inviter les itinéraires de découverte et l'option de découverte professionnelle en $3^{\mathrm{e}}$ ? L'apprentissage d'une langue vivante étrangère peut-il, dans un établissement scolaire, comprendre un temps prolongé d'« immersion » initiale, au lieu des deux ou trois séquences horaires hebdomadaires traditionnelles? Les regroupements par groupes de besoin sont-ils fréquents? Comment s'organise le soutien individualisé en $6^{\mathrm{e}}$ par exemple? Tombe-t-on dans l'ornière facile des simples dédoublements? L'hétérogénéité des classes, fixée comme un objectif, est-elle respectée dans la constitution des groupes? La pression locale ne pousse-t-elle pas à des regroupements par niveaux, et à la juxtaposition de classes aux ambitions et attentes très différentes?

4 L'autonomie, et l'horizon important qu'elle pourrait découvrir, peuvent être facilement bridés par les habitudes et les "intérêts" acquis des différents acteurs. Casser les schémas suppose une volonté forte et le soutien d'un assentiment partagé...

\section{Les outils actuels de l'autonomie}

5 La loi d'orientation de 1989 suggère que l'autonomie prenne corps dans le «projet d'établissement » qui définit les modalités de mise en œuvre des programmes nationaux et des orientations nationales et académiques; il assure la cohérence des actions au service des élèves. Il sera d'autant plus traduit dans les faits que la cohérence des interventions des différents personnels de l'établissement y est recherchée, ainsi que l'ouverture aux parents et à différents partenariats. Au cœur de l'activité pédagogique, le projet d'établissement exprime des choix concertés, formule des priorités, dans une démarche globale où s'exprime la responsabilité du chef d'établissement.

6 Ce projet peut prévoir le recours à des procédures contractuelles qui visent à organiser les relations de l'établissement avec l'autorité académique, dans le respect de l'espace d'initiative et d'adaptation consenti par l'État. Cette contractualisation précise les objectifs assignés à l'établissement, après concertation avec le conseil d'administration. Il n'y a pas de contrôle a priori de pertinence et d'opportunité pour le projet d'établissement: un "simple " contrôle de légalité quant à la mise en œuvre des objectifs nationaux est assuré. L'autorité académique élabore des analyses partagées, contractualise des engagements et des garanties réciproques. Une approche globale des moyens attribués, mise en œuvre de façon annualisée par certains départements, est l'un des aspects de cet engagement.

7 Il revient à l'autorité académique d'assurer un conseil, de mettre en place des dispositifs d'accompagnement des établissements scolaires, en veillant à leur inscription dans la durée (de trois à quatre ans). C'est donc l'inverse de l'indépendance.

8 Pour donner à la communauté éducative les moyens de sa mission, le règlement intérieur en définit les règles de fonctionnement, les droits et obligations de chacun de ses membres. Il exprime de façon explicite les valeurs partagées par la communauté scolaire et incite au respect de la discipline, organise une vie scolaire riche, deux facteurs propices à réduire l'absentéisme et autoriser la réussite des élèves.

9 Le projet de budget participe de la déclinaison pratique des orientations retenues par cette communauté spécifique. 
10 Le rapport d'activités, corollaire de l'autonomie de l'établissement, rend compte des actions conduites au service de la réussite des élèves, mesure et rend publics les résultats. Il peut attirer l'attention des différents acteurs sur les nécessaires régulations et contribue à créer un espace de sens partagé.

\section{Le pilotage}

Que retenir comme outils de cette mise en tension « du centre vers la périphérie »?

- Sans doute la mise en œuvre de la circulaire de 1997 sur les missions du professeur, élément actif d'une communauté éducative, et non pas "spécialiste» disciplinaire seulement, dispensateur d'un savoir abstrait; souvent, il est reproché aux professeurs d'exercer une "profession libérale", hors contrôle! Le corollaire en est une inspection pédagogique capable d'intervenir, au-delà de sa seule expertise disciplinaire, sur des aspects éducatifs généraux. L'inflexion est prise, l'ESEN œuvre à la rendre effective.

- Le recentrage des enseignants sur des exigences clairement exprimées en termes d'objectifs à atteindre ; la formation continue et en particulier un plan annuel de formation continue de l'établissement paraissent conditionner l'atteinte de cet objectif.

- Un pilotage par les résultats des élèves, mesurés de façon « extérieure » à l'établissement. Sans doute convient-il de développer, à l'échelle nationale et académique, la mise à disposition d'indicateurs de performance, en particulier pour le public des collèges.

- Un recrutement qualitatif des chefs d'établissement. Le chef, porteur des valeurs de l'école républicaine, doit être capable d'assurer un pilotage de l'établissement en conjuguant la compétence pédagogique générale, la capacité à manager, à communiquer, à rendre compte, à être responsable. Le ministère est en train de définir et décliner une nouvelle politique de recrutement, de renouveler les modalités du concours en particulier. L'ESEN ouvre par ailleurs la formation des personnels d'encadrement aux exigences managériales des autres grandes écoles de la fonction publique française.

12 Les chefs d'établissement nous ont habitués à réclamer toujours plus d'autonomie. Notamment en matière de recrutement et de gestion des personnels, les chefs d'établissement se plaignent le plus souvent de ne pas pouvoir recruter librement leur adjoint, leurs professeurs, etc. Or le premier pas consenti vers ce recrutement par l'EPLE, celui des assistants d'éducation, a soulevé en 2003 la contestation dans l'ensemble du pays. Les programmes disciplinaires, nationaux, considérés généralement comme trop chargés, sont cependant farouchement défendus... L'autonomie, selon la récente expression de chefs d'établissement, "c'est un état d'esprit ». Ce n'est pas un but en soi, c'est une recherche de sens, «c'est l'espace de sens que nous allons nous donner après accomplissement de toutes les injonctions ».

\section{Autonomie et décentralisation}

Dans la tension actuelle entre les orientations ministérielles nationales et l'adaptation aux réalités locales, l'établissement scolaire français doit ajouter un troisième pôle, dont le poids s'accroît soudain dans cette nouvelle phase de décentralisation, celui des collectivités territoriales, régionales ou départementales. Depuis l'automne dernier et le transfert annoncé des personnels TOSS, de nombreuses manifestations d'inquiétude se font jour. Même la mise à disposition de manuels scolaires à titre gratuit dans les 
lycées à l'automne 2004 a pu être ressentie « de façon schizophrénique ». Que dire du « ressenti » lorsqu'il s'accompagne ici ou là d'une lettre du président de la collectivité au seul «président du Conseil d'administration », et appelle ce même personnage, par ailleurs représentant de l'État, à lire en séance un courrier reprochant à l'État telle ou telle orientation? Selon les cas, le chef d'établissement français est renvoyé à une forme de dédoublement schizophrénique impossible à tenir.

Un équilibre politique nouveau est à atteindre, qui se cherche encore en ce moment où tous les aspects du transfert des personnels TOSS ne sont pas résolus.

Ce troisième pôle relatif au poids des collectivités territoriales, plus fort qu'il n'avait été dans la première phase de décentralisation des années 1980, appelle justement des chefs d'établissement plus responsables encore, capables d'analyse des enjeux, de mise à distance ; des chefs d'établissement qui pilotent une communauté en lui donnant des caps, dans une recherche de sens qu'elle partage, où l'échelon académique l'accompagne et la soutient. Le "pilotage pédagogique partagé » déjà en œuvre entre inspecteurs territoriaux et chefs d'établissement est plus que jamais d'actualité.

L'autonomie de l'établissement scolaire est un facteur d'efficacité pédagogique et de réussite des élèves, à conjuguer avec la responsabilité du chef d'établissement, dans le cadre d'une lettre de mission, et le soutien d'une autorité académique qui contractualise.

INDEX

Index géographique : France

\section{AUTEUR}

\section{CÉCILE BRENNAN-SARDOU}

Inspectrice d'académie adjointe des Alpes-Maritimes. 